\title{
Vortex Method of Localization of Thermonuclear Plasma
}

\author{
Eugeniy Pavlovich Smirnov \\ Independent Researcher, Moscow, Russia \\ Email: techofculture@narod.ru
}

How to cite this paper: Smirnov, E.P. (2017) Vortex Method of Localization of Thermonuclear Plasma. Journal of Power and Energy Engineering, 5, 58-65. https://doi.org/10.4236/jpee.2017.512008

Received: October 19, 2017

Accepted: December 11, 2017

Published: December 14, 2017

Copyright (C) 2017 by author and Scientific Research Publishing Inc. This work is licensed under the Creative Commons Attribution International License (CC BY 4.0).

http://creativecommons.org/licenses/by/4.0/

\begin{abstract}
An important task of world energy is to solve the problem of controlled thermonuclear fusion and the information presented in the article can be used in power engineering to create a controlled thermonuclear reactor. The method now used magnetic localization of plasma does not allow sustaining stable thermonuclear plasma in a closed chamber, and so another solution to the problem is necessary. In this paper, we propose an alternative dynamic approach of the stationary localization of plasma through centrifugal force. Localization of plasma as plasma whirlwind allows us to control the process of stable thermonuclear fusion.
\end{abstract}

\section{Keywords}

Controlled Thermonuclear Fusion, Thermonuclear Plasma, Centrifugal Force, Plasma Whirlwind

\section{Introduction}

"The first important task of physics in the 21st century remains solution of the problem of controlled thermonuclear fusion". V. L. Ginzburg (Problems of Physics of the 21st Century. Speech in Kurchatov Institute of 2002).

Many years have passed since the task of realizing controlled thermonuclear fusion was set. The author of the pioneer research program for controlled thermonuclear fusion was Spitzer L. [1]. Since then, the methods of magnetic confinement of high-temperature thermonuclear plasma in magnetic traps of tokamak type and stellarator have been used [2].

Tokamak was proposed in the USSR [3]. The principle on which he works is simple. Plasma is retained by a magnetic field, which is created by a toroidal solenoid. The plasma is under pressure several atmospheres. The current flowing 
in the plasma creates its own a magnetic field that compresses the plasma into a cord. The plasma has the form of a torus and is enclosed within a solenoid, in which creates a magnetic field. As a result, it turns out that the plasma is surrounded by vacuum insulation. This is necessary to maintain plasma at high temperatures, at which a thermonuclear reaction occurs.

The idea of the stellarator was put forward by Spitzer L. In a stellarator on a closed toroidal magnetic field additional field is added, created by a special screw the winding wound on the body of the reaction chamber. The main feature of the stallarator is that the applied in it the method of confinement of plasma is not connected with the presence of a current in the plasma, as in a tokamak.

Studies are known of the inertial controlled thermonuclear fusion without the use of magnetic confinement of plasma [3]. The idea of the method is that pulsed heating occurs mixture of deuterium and tritium in the frozen state approximately millimeter diameter in a very short period of time, for which the clot does not have time to scatter. This creates a very high pressure, which ensures intensive heat exchange between ions and electrons of the thermonuclear plasma. It is believed that in this way the thermonuclear $\mathrm{D}+\mathrm{T}$-reaction in the clot can be almost completely completed. This requires a well-focused highpower laser radiation, which must heat the clot simultaneously and with all sides for a time of the order of nanoseconds. In laser thermonuclear fusion experimentally achieved plasma temperature $8 \times 10^{7} \mathrm{~K}$. The appearance of neutrons confirms the principle possibility initiate a thermonuclear reaction in this way. However, a relatively small number of them call into question such a mechanism for confinement and combustion of thermonuclear fuel on all its volume.At present, JET and JT-60U tokamaks are obtained the power of a thermonuclear reaction comparable to the power of the creation of a plasma, and on this basis the international tokamak-reactor ITER is being designed. However, before practical application controlled thermonuclear fusion based on the confinement of thermonuclear plasma by a magnetic field far away, since such characteristics of the reactor, such as efficiency, manufacturability, the price of electricity received, and environmental friendliness are at the forefront.

The purpose of this paper is to propose a alternative method for retaining thermonuclear plasma, in which the plasma is not confined by a magnetic field, but centrifugal force is isolating high-temperature plasma from the chamber wall in which a plasma is formed, than is provided the possibility of implementing active control actions on the process of occurrence and steady thermonuclear synthesis reaction.

\section{Statement of the Problem}

Until now, people used energy to get energy processes of terrestrial origin observed in natural conditions, for example, burning, the force of the falling water, wind power and so on. Synthesis of light nuclei at a very high temperatures are widespread in nature, but not on the Earth, but in the bowels of stars, where matter is in plasma state. Such reactions include the formation of helium from 
hydrogen, taking place, according to modern representations, inside the Sun at a temperature of about 20 million degrees. It is this reaction that keeps the temperature of the Sun. To release this energy in the form of a relatively peaceful burning, suitable for peaceful consumption-in this is the problem of controlled thermonuclear fusion.

What are the causes of those difficulties on the way that nature infinitely long time and successfully implements in super-scales? Why physicists brought the controlled thermonuclear fusion at number 1 to the list of their problems by degree of difficulty and importance?

In nature, the plasma is retained by gravitational forces. Instead of gravity to hold the plasma, it was suggested to use magnets. Plasma plants built in which magnetic lines of force had the most complex configurations-"Bottles", "plugs", "mirrors", "eight",-really kept the plasma in their invisible boundaries, but only of too short a time of the order of microseconds. It turned out that the reason for this-the instability of plasma formations, which this method did not allow to control [2]. Is there another way?

\section{New Way}

The new way consists in the refusal of preferential use magnetic field for confinement of thermonuclear plasma. The magnetic method is static and does not provide steady-state retention plasma, because he does not allow you to effectively manage the process. Instead, alternative method of plasma confinement by centrifugal force offered. It is possible to carry out active control actions on the process of the formation and flow of a thermonuclear fusion reaction.

The powerful gravitational field of a star, for example, the Sun, does not give the bulk mass of heated to very high temperatures hydrogen to escape into outer space. Can not go and the reaction products of synthesis, releasing heat and warming upother particles. It is believed that such a regime of self-heating and plasma self-control can not be realized on the Earth.

However, the behavior of the body in an accelerated moving system is equivalent to the behavior of the body in the presence of an equivalent gravitational field. This is a qualitative consideration lies at the basis of Einstein's general theory of relativity.

\section{Suggestion}

So, on the Earth, you can organize such an accelerated the motion of the system, which by action on the body is equivalent to the gravitational field. Such accelerated motion can be a uniform rotation of the system, which creates in the system the centrifugal force of inertia and which can be made to serve to hold the plasma. In such a natural way, we come to the necessity use of the concept of a vortex.

The beginning of the modern theory of vortex motion was based G. Helmholtz, published in 1858 his memoir "About integral of the hydrodynamic equa- 
tions corresponding to vortex motion "[4], in which he first formulated a theorem on the conservation of vortices. According to this theorem for forces satisfying the conservation law energy, it is impossible to create or destroy existing vortex. Only after the work of Helmholtz arose a group of issues and tasks that now constitute the subject of the doctrine of vortices.

The development of the proposed concept can be helped by observations and studies of tornadoes [5].

TWIRLLARATOR - a new term derived from the word TWIRL. Figure 1 shows schematically the TWIRLLARATOR, which consists of the reaction chamber 1, the lower gas circuit where the gas can flow with the possibility of changing its temperature and pressure, 2 and the flap 3 separating them. A linear gas vortex 4 is formed in the reaction chamber. A electromagnetic system for initiating and maintaining high temperature plasma in the reaction chamber 5 , for this it is possible to use other known means.

In tokamaks ions are retained in a limited volume by a strong magnetic field. The heating process is performed by electrons, which are initially short current pulses are heated to very high temperatures Then, through Coulomb collisions, electrons transfer their energy ions. In the conditions adopted in modern tokamak projects, the time, for which electrons transfer their energy to ions, reaches 20 - 30 seconds. It turns out that during this time most of the electron energy will go away in bremsstrahlung. Therefore, in modern tokamaks additional sources of plasma heating are used. In the twirllarator can also be used additional more effective methods of supplying energy to ions [3]. This can be either high-frequency heating, or injection of fast neutral deuterium atoms, or dissipation of magnetoacoustic waves [6].

The picture of the phenomena in the TWIRLLARATOR

1) The lower gas circuit and the reaction chamber are filled with gas (for example, a mixture of Dt and Tr). The flap closes. Turn on the rotation of the system and achieve steady rotation of the gas vortex in the reaction chamber.

When the cylindrical reaction chamber rotates with the gas, the inner adjacent

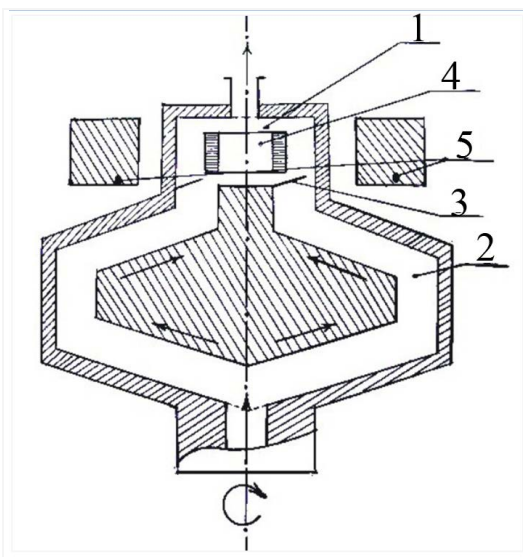

Figure 1. Schematic image of the twirllarator. A source: https://plus.google.com/communities/104524269775067972384 
gas layer is entrained by the chamber and begins to participate in the rotational motion. Near the inner surface of the rotating cylinder, the gas layer acquires over time almost the same linear velocity as the velocity of the points on the inner surface of the cylinder of the reaction chamber. Due to internal friction, the moment of momentum is transferred to adjacent gas layers and eventually the motion captures the entire internal area of the reaction chamber. A linear gas vortex is formed. Centrifugal forces create a pressure gradient and, as a result, the system is held in balance.

Unlike liquid vortices whose center is filled with a liquid of the same density as their periphery, the gas vortex has a tubular structure with a compacted wall in which the bulk of the gas vortex is located [3]. In the central part of the gas vortex, the gas pressure is reduced by scattering of particles from the center by centrifugal force, and the vortex itself is bounded from the outside by a boundary layer with reduced values of temperature and viscosity and with values of the rotational speed smoothly changing from the speed of the outer wall of the vortex to the velocity of the rotating wall of the reaction chamber.

2) The cooled gas is supplied to the lower gas circuit. It is possible to vary the temperature and pressure of the gas. The flap opens. There is a secession of the vortex from the walls of the chamber and its compression. The velocity of the vortex increases

If the external gas pressure acts on the gas vortex on its different sides, this exerts a compressive effect on the vortex as an integral structure. Compression of the body of the vortex by external forces caused by ambient pressure causes an increase in the rotation speed of the wall of the gas tube of the vortex, and the internal pressure decreases.

In the boundary layer around the vortex, the gradients of velocities, pressures and temperatures will be large and the viscosity and thermal conductivity of the gas are greatly reduced. Under such conditions, ring motion and heat are almost not transferred to the outer layers of the boundary layer. This state of the vortex will be stable. The wall of the gas vortex will rotate in the boundary layer, as in a sliding bearing, almost without transferring of movement and heat.

3) Turn on the current in the electromagnetic system near the reaction chamber. Gas breakdown in the wall of the gas vortex is achieved. The gas is ionized and the current flowing through it heats it to a high temperature. It is also possible to use other known means for the initialization and maintenance of hightemperature plasma.

When the system rotates, the centrifugal force causes the cold gas to press against the walls of the lower gas circuit. The pressure of the cold gas in the lower expanded circuit, transferred to the narrow and hot reaction chamber, will overpower the pressure from the centrifugal force of the light ionized gas in the narrow reaction chamber.

By changing the radius of the lower gas loop, when designing the device, the system rotation speed, the temperature and the pressure of gas supplied to the lower gas circuit, the high temperature Plasma Whirlwind of the desired tem- 
perature is formed in the reaction chamber and its stable rotation.

\section{Conclusions}

The main obstacle in solving the problem of controlled thermonuclear fusion now-not yet deep enough physical processes in hot plasma have been studied. The theory, which is well developed here, refers only to non-turbulent state of the plasma.

In a plasma the mean free path of both electrons and ions reaches centimeters, and the temperature gradient is large. Therefore, the internal stress, according to Maxwell [8], approximately 10 orders of magnitude more than in gas, and can create in plasma convective flows and turbulence. The hot plasma is in a turbulent state. Even in ordinary hydrodynamics turbulent processes do not have full quantitative description and basically all calculations are based on the theory of similarity. In a plasma hydrodynamic processes are more complex, so it is necessary to go the same way.

The main obstacle in the construction of the twirllarator lies in the inevitable engineering problems.

As practically in all nuclear reactors, the generated power is proportional to the volume of the core and the losses increase only with an increase in its surface, the efficiency of nuclear reactors grows with the growth of their size. Therefore, the reactor has a critical size, from which it can generate useful energy. The magnitude of this critical size determines the feasibility reactor in practice. This critical size is determined not by scientists, but by engineers-designers, since it is closely related to the constructive design of the whole installation as a whole. Life shows that the inventive abilities of people do not have limit, and therefore the critical dimensions of twirllarator can with time become feasible. The method used in tokamak and stellarator is now amenable full theoretical calculation, but the building of a thermonuclear reactor, based on magnetic confinement of plasma, leads to a very large and complex construction. In contrast, twirllarator has a simple construction. But its practical implementation and dimensions depend on convection heat exchange processes that are not amenable to theoretical quantitative assessment. Experiments are necessary.

The main attraction of scientific work is that it leads to problems, the solution of which can not be foreseen, therefore the solution of problem of controlled thermonuclear fusion for the scientist especially attractive.

\section{Acknowledgements}

In [7] described experiments with phenomena similar to phenomena in the twirllarator. In these experiments a hot plasma was obtained. Through the gas missed the radiation from the powerful high-frequency generator of continuous operation. At the same time arose a free-floating gas discharge oval shape.

The plasma discharge had the shape of a cord about 10 centimeters, equal to 
half the wavelength of high-frequency oscillations. The plasma pinch was in one of the maxima of electric field. Stability of the plasma pinch along the longitudinal axis was created by electric field of high-frequency oscillations. In the radial direction the stability of the plasma pinch was provided by the rotation of the gas.

Of greatest interest is the study of plasma discharge in hydrogen or in deuterium. At low powers the discharge did not have clearly delineated boundaries. His glow was of a diffuse nature. With an increase in the applied high-frequency oscillations power the radiation became brighter, the diameter of the discharge increased and inside appeared the clearly defined core of the cord form, the cross section of which grew with power input. The higher was the pressure, the more was stable of the discharge, and the sharper was forms of core. Studying the conductivity of the plasma, and also with the help of active and passive spectral diagnostics of plasma it could be reliably established that the central part of the discharge had a very high temperature of electrons-above a million degrees. Thus, at the boundary of the plasma cord, at a distance of several millimeters, there was a temperature gradient of over one million degrees. This meant that the plasma on the surface of the cord had a high thermal insulation. The possibility of such a large temperature gradient initially caused doubts. Therefore, various methods of plasma diagnostics were tested. But all of them invariably led to the same high temperature-above a million degrees. But later it became clear that the physical nature of existence such a temperature gradient is quite understandable. Such a large temperature gradient can be explained by the fact that on the boundary of a hot plasma there is a double electric layer, from which without significant losses electrons are reflected. The possibility of the existence of such a layer has long been known.

These experiments confirm that it is possible to try to implement the project of twirllarator-the thermonuclear reactor to obtain useful energy.

\section{References}

[1] Spitzer, L. (1962) Physics of Fully Ionized Gases. 2nd Edition, Interscience, New York.

[2] Arcimovich, L.A. (1963) Upravljaemye termojadernye reakcii. Nauka, Moscow.

[3] Ribe, F.L. (1975) Fusion Reactor Systems. Reviews of Modern Physics, 47, 7. https://doi.org/10.1103/RevModPhys.47.7

[4] Helmholtz, G. Wissenschaftliche Abhandlungen, Bd 1-3, Leipzig, 1882-1895; Vorlesungen uber theoretische Physik, Bd 1-6, Leipzig, 1898-1903.

[5] Smirnov, E.P. (2016) Numerical Study of Hydrodynamics and Heat and Mass Transfer of Two-Phase Flow in Atmospheric Tornado-Forming Cloud and a Tornado Model. High Temperature, 54, 282-289. https://doi.org/10.1134/S0018151X1602019X

[6] Kapica, P.L. and Pitaevskij, L.P. (1974) Nagrev plazmy magnitoakustiskimi kolebanijami. ZhJeTF, t.67, s.1410.

[7] Kapica, P.L. (1969) Svobodnyj plazmennyj shnur v vysokochastotnom pole pri vy- 
sokom davlenii. ZhJeTF, t.57, s.1801.

[8] Maxwell, J.C. (1879) On Stresses in Rarified Gases Arising from Inequalities of Temperature. Philosophical Transactions of the Royal Society, 170, 231.

https://doi.org/10.1098/rstl.1879.0067 